\title{
A Small GTPase RHO2 Plays an Important Role in Pre-infection Development in the Rice Blast Pathogen Magnaporthe oryzae
}

\author{
Teng Fu', Joon-Oh Kim ${ }^{1}$, Joon-Hee Han ${ }^{1}$, Adiyantara Gumilang', Yong-Hwan Lee ${ }^{2}$, and Kyoung Su Kim (D) ${ }^{1 *}$ \\ ${ }^{I}$ Division of Bioresource Sciences, and Bioherb Research Institute, Kangwon National University, Chuncheon 24341, \\ Korea \\ ${ }^{2}$ Department of Agricultural Biotechnology, and Center for Fungal Genetic Resources, Seoul National University, Seoul \\ 08826, Korea
}

(Received on April 18, 2018; Revised on August 17, 2018; Accepted on August 21, 2018)

The rice blast pathogen Magnaporthe oryzae is a global threat to rice production. Here we characterized $\mathrm{RHO} 2$ gene (MGG_02457) that belongs to the Rho GTPase family, using a deletion mutant. This mutant $\triangle M$ Morho 2 exhibited no defects in conidiation and germination but developed only $6 \%$ of appressoria in response to a hydrophobic surface when compared to the wild-type progenitor. This result indicates that $\mathrm{MoRHO} 2$ plays a role in appressorium development. Furthermore, exogenous cAMP treatment on the mutant led to appressoria that exhibited abnormal morphology on both hydrophobic and hydrophilic surfaces. These outcomes suggested the involvement of $\mathrm{MoRHO2}$ in CAMP-mediated appressorium development. $\triangle M$ Morho 2 mutation also delayed the development of appressorium-like structures (ALS) at hyphal tips on hydrophobic surface, which were also abnormally shaped. These results suggested that MoRHO2 is involved in morphological development of appressoria and ALS from conidia and hyphae, respectively. As expected, $\triangle M$ Morho2 mutant was defective in plant penetration, but was still able to cause lesions, albeit at a reduced rate on wounded plants. These results implied that $\mathrm{MoRHO} 2$ plays a role in M. oryzae

\footnotetext{
*Corresponding author.

Phone) +82-33-250-6435, FAX) +82-33-259-5558

E-mail)kims@kangwon.ac.kr

ORCID

Kyoung Su Kim

http://orcid.org/0000-0002-0827-0844

(c) This is an Open Access article distributed under the terms of the Creative Commons Attribution Non-Commercial License (http:// creativecommons.org/licenses/by-nc/4.0) which permits unrestricted noncommercial use, distribution, and reproduction in any medium, provided the original work is properly cited.
}

Articles can be freely viewed online at www.ppjonline.org. virulence as well.

Keywords : appressorium formation, Magnaporthe oryzae, pathogenicity, Rho GTPase

Handling Associate Editor : Shim, Won Bo

Magnaporthe oryzae, an ascomycete plant pathogenic fungus, causes severe loss of rice production every year that is equivalent to the amount that can feed 60 million people (Han et al., 2015; Kim et al., 2009; Talbot et al., 1993). Utilization of suitable resistant cultivars is considered an effective strategy to control rice blasts. However, M. oryzae overcomes host resistance within several years after their initial appearance in rice paddy fields (Saleh et al., 2014; Zhu et al., 2017). The rice blast caused by $M$. oryzae starts when germ tubes of conidia emerge on rice leaves (Hamer and Talbot, 1998). Subsequently, a domeshaped specialized infection structure called appressorium develops on the tip of the germ tube before the rice cuticle is penetrated (Dean, 1997; Ryder and Talbot, 2015). The buildup of turgor pressure (up to $8 \mathrm{MPa}$ ) inside the appressorium is a key prerequisite for penetration of plant cell walls (Dean et al., 2005). After successful invasion and colonization, asexually reproduced conidia disseminate to uninfected plants. M. oryzae is a polycyclic pathogen and is able to infect almost all tissues of rice plants, which accounts for the severe loss of rice production during favorable conditions (Han et al., 2015; Kim and Lee, 2012).

Regulation of morphogenesis is essential for successful pathogenic development in fungi. The Rho protein family, which was first identified in Aplysia species, belongs to the family of small GTPases (Madaule and Axel, 1985; Ridley, 
2001). Most Rho proteins are GTP-hydrolyzing molecular switches, which participate in different signaling pathways for cell growth, morphogenesis, and gene regulation (Arellano et al., 1999; Etienne-Manneville and Hall, 2002). Rho GTPases contain five highly conserved motifs (G1-G5) and two functional elements, switch I between G1 and G2 and switch II between G2 and G3 (Smithers and Overduin, 2016). The G1-G5 loops form nucleotide-binding sites, regulating GTP hydrolysis (Hanna and El-Sibai, 2013). The switch I element is a core effector that is important for the interaction with downstream partners (Karnoub et al., 2004). The switch II element coordinates nucleophilic water that is required for GTPase activity (Schaefer et al., 2014). A special helical amino acid sequence of approximately 10-15 residues located between G4 and G5 distinguishes the Rho protein family from other GTPases. This helical sequence has no effect on the function of GDP or GTP binding but modulates Rho protein activity through an interaction with guanine nucleotide exchange factors, thus affecting downstream effectors (Wittinghofer and Vetter, 2011).

In the fungal kingdom, Rho proteins modulate diverse biological processes such as growth, differentiation, secretion, vesicular trafficking, and transcription. Several members of the Rho family have been characterized in fungal pathogens, including $\mathrm{RHO}$, $\mathrm{RHO} 2, \mathrm{RHO} 3$, RHO4, CDC42, and RACl (Dean et al., 2005; Du et al., 2013). RHO1 gene helps control cell wall synthesis and aggregation of actin cables in Fusarium oxysporum, Aspergillus nidulans, and Saccharomyces cerevisiae (Guest et al., 2004; Martínez-Rocha et al., 2008; Ridley, 2001). RHO2 is involved in regulating the cell wall integrated pathway in Neurospora crassa and Colletotrichum gloeosporioides (Richthammer et al., 2012; Xu et al., 2016). $\mathrm{RHO} 3$ is associated with the regulation of mitochondria distribution, conidial morphology, germination, and vegetative growth in M. oryzae, Trichoderma reesei, and Botrytis cinerea (An et al., 2015; Vasara et al., 2001; Zheng et al., 2007). RHO4 is involved in septa formation by regulating the actin cytoskeleton in fission yeast and $N$. crassa (Nakano et al., 2003; Rasmussen and Glass, 2005). In Ustilago maydis, CDC42 serves as an effector of cell separation during budding, and $R A C l$ is necessary for the switch from budding to hyphal growth (Mahlert et al., 2006). However, the detailed functions of $\mathrm{RHO}_{2}$ in M. oryzae have not been identified.

In this study, we functionally characterized $\mathrm{MoRHO2}$ using a targeted gene deletion in M. oryzae. Our results indicate that deletion of $\mathrm{MoRHO} 2$ does not affect vegetative growth, conidiation, or conidial germination, but it leads to a delayed development of appressoria on hydrophobic surfaces. Exogenous cAMP treatment only partially rescues morphologically abnormal development of appressoria. Taken together, our data suggest that MoRHO2 plays an important role in pre-infection development in M. oryzae.

\section{Materials and Methods}

Fungal strains and culture conditions. The wild type (KJ201) and transformants obtained in this study were grown on medium that included oatmeal $(50 \mathrm{~g} / \mathrm{l}$ oatmeal and $25 \mathrm{~g} / \mathrm{l}$ agar powder), complete medium (6 g/l yeast extract, $6 \mathrm{~g} / \mathrm{l}$ casamino acids, $10 \mathrm{~g} / \mathrm{l}$ sucrose, and $15 \mathrm{~g} / \mathrm{l}$ agar powder) and V8 medium ( $80 \mathrm{ml} \mathrm{V8} \mathrm{juice,} 310 \mu 110 \mathrm{~N}$ $\mathrm{NaOH}$ solution, and $15 \mathrm{~g} / \mathrm{l}$ agar powder) at $25^{\circ} \mathrm{C}$ with constant light. The conidial suspension was prepared by filtrating conidia obtained from 7-day-old V8 medium through two layers of Miracloth (Calbiochem, San Diego, CA, USA). Mycelia grown in liquid conditioned medium (CM) for 8 days were used to extract DNA and RNA.

Sequence and phylogenetic analysis. We obtained all sequences from online databases of the Broad Institute of MIT and Harvard (http://www.Broadinstitute.org), the National Center for Biotechnology Information (http://www. ncbi.nlm.nih.gov), and the Comparative Fungal Genomics Platform (http://cfgp.riceblast.snu.ac.kr) (Park et al., 2007). MEGA 6.0 was used to analyze phylogenetic relationship of MoRHO2 with orthologs of other organisms. Domain structures of the Rho protein family were analyzed with InterPro Scan, version 53 (http://www.ebi.ac.uk/interpro/), and visualized using Illustrator for Biological Sequences, version 1.0.3. All primers in this study were designed by Primer Quest Design Tool (http://sg.idtdan.com/site).

RNA isolation and gene expression analysis. The RNA (5 $\mu \mathrm{g})$ used to synthesize cDNA, was extracted from mycelia, conidia, germinated conidia, appressoria and invasive hyphae in rice leaves, by using Easy-Spin ${ }^{\mathrm{TM}}$. RT-PCR was conducted to detect transcripts of $\mathrm{MoRHO} 2$ in transformants with RTF/RTR primers (Han et al., 2015). The process was performed in a $20-\mu 1$ mixture containing $1 \mu 1$ cDNA $(25 \mu \mathrm{g} / \mu \mathrm{l}), 1 \mu \mathrm{l}$ forward primer, $1 \mu \mathrm{l}$ reverse primer, $4 \mu \mathrm{l}$ Pfu polymerase, and $13 \mu \mathrm{l}$ sterilized distilled water. In total, 30 cycles of RT-PCR were run in an Applied Biosystems 2720 Thermal Cycler (Applied Biosystems, Foster City, CA, USA). qRT-PCR was performed to compare the expression levels of $\mathrm{MoRHO} 2$ during development of $M$. oryzae as previously described (Han et al., 2015). The reaction was performed using a $10-\mu 1$ mixture containing $1 \mu \mathrm{l}$ 
cDNA, $1 \mu 1$ forward primer, $1 \mu 1$ reverse primer, and HIPI Real-Time PCR $2 \times$ Master Mix (SYBR Green; Molecular Probes, Eugene, OR, USA). PCR was performed with qRTF and qRTR primers using the One Step Real-Time PCR System (Applied Biosystems). Cycle threshold (Ct) was normalized to that of the $\beta$-tubulin gene (MGG00604) and treated samples as $2^{-\Delta C t}$, where $-\Delta C t=\left(\mathrm{C}_{\mathrm{t}}\right.$, target gene $\mathrm{C}_{\mathrm{t}, \beta \text {-tubulin }}$ ). Fold changes in expression were calculated as $2^{-\Delta \Delta C t}$, where $-\Delta \Delta C t=\left(\mathrm{C}_{\mathrm{t}} \text {, target gene }-\mathrm{C}_{\mathrm{t}, \beta \text {-tubulin }}\right)_{\text {test condition }}-\left(\mathrm{C}_{\mathrm{t}}\right.$, $\left.{ }_{\mathrm{WT}}-\mathrm{C}_{\mathrm{t}, \beta \text {-tubulin }}\right)_{\mathrm{CM}}$. Three replicates were conducted to confirm the results of qRT-PCR.

Targeted deletion of $\mathrm{MoRHO} 2$ and construction of complementation strains. MoRHO2 targeted deletion construct was generated by double-joint PCR (Choi et al., 2011; Yu et al., 2004). Upstream and downstream flanking regions $(1.5 \mathrm{~kb})$ of the target gene were amplified by primers $3 \mathrm{~F} / 3 \mathrm{R}$ and $5 \mathrm{~F} / 5 \mathrm{R}$ (Table 1 ). The $H P H$ cassette was amplified using primers HPH-F/HPH-R. The three PCR products were fused with primers $5 F / 3 R$. The final

Table 1. List of primers used in this study.

\begin{tabular}{|c|c|}
\hline Primer & Sequence $\left(5^{\prime} \rightarrow 3^{\prime}\right)$ \\
\hline \multicolumn{2}{|l|}{ MoRHO2 } \\
\hline $5 \mathrm{~F}$ & TCATGACGAGTCGGCAATCACT \\
\hline $5 \mathrm{R}$ & $\begin{array}{l}\text { CCTCCACTAGCTCCAGCCAAGCCATGT- } \\
\text { GCTTGCTGCTGATGCTGT }\end{array}$ \\
\hline $3 \mathrm{~F}$ & $\begin{array}{l}\text { GTTGGTGTCGATGTCAGCTCCGGAGGC- } \\
\text { GTTCTTCTACGACATCTGACGA }\end{array}$ \\
\hline $3 \mathrm{R}$ & ACACATCAAGGCAACGCTGA \\
\hline NF & AAGCAAACAGTTCCAGGGCA \\
\hline NR & GGCATCAGCATCAACCTTTA \\
\hline SF & AGTCAACGGACCAACCCTTT \\
\hline SR & AAAGGAGCATCGAGGTCTGT \\
\hline RTF & GTCGGTGCAGCTAGCATTAT \\
\hline RTR & TAGCCTCTTCGATCCACTATGA \\
\hline $\mathrm{cmF}$ & ACGAATCTGCACCACATCAGGA \\
\hline $\mathrm{cmR}$ & CGCACATGTTTGTTTCGTCGTC \\
\hline PF & AAGCAAACAGTTCCAGGGCAATCG \\
\hline PR & ATCTGCAGTCGGCTGGTGTAAAGA \\
\hline qRTF & AGGACTACGAGCGATTAC \\
\hline qRTR & GATCCACTTGTGCTTCAC \\
\hline \multicolumn{2}{|c|}{ Hygromycin phosphotransferase } \\
\hline HPH_F & GGCTTGGCTGGAGCTAGTGGAGG \\
\hline HPH_R & CTCCGGAGCTGACATCGACACCAAC \\
\hline \multicolumn{2}{|l|}{$\beta$-tubulin } \\
\hline$\beta$-tubF & TCGACAGCAATGGAGTTTAC \\
\hline$\beta$-tubR & AGCACCAGACTGACCGAAGAC \\
\hline
\end{tabular}

deletion construct was amplified by primers NF/NR, and subsequently transformed into wild-type protoplasts (Kim et al., 2009). Hygromycin-resistant transformants were selected on TB3 medium containing Hygromycin B (200 $\mu \mathrm{L} / \mathrm{ml}$ ). Southern blotting and RT-PCR were used to confirm the targeted deletions. For complementation, the targeted gene was amplified from the wild-type genome by primers $\mathrm{cmF} / \mathrm{cmR}$. The amplicon was used to co-transform protoplasts of the deletion mutant with the pII99 vector containing a Geneticin-resistance cassette.

Appressorium formation on the hydrophobic surface. Conidia grown on 6-day-old V8 medium were harvested by adding $5 \mathrm{~mL}$ sterilized, distilled water and filtering through two layers of Miracloth (Calbiochem). The conidial suspension was centrifuged at 5,000 rpm for $10 \mathrm{~min}$, followed by removal of the supernatant, and was diluted to a final concentration of $5 \times 10^{4}$ conidia $/ \mathrm{ml}$ (Han et al., 2015; Kim et al., 2014). Drops of conidial suspension (20 $\mu 1)$ were placed on hydrophobic coverslips and hydrophilic slide glasses with or without the addition of exogenous $5 \mathrm{mM}$ cAMP and incubated in moist boxes. Appressoria formed on germinated conidia were calculated by counting at least 100 conidia per replicate in at least three independent experiments with three replicates per experiment.

Wound inoculation and sheath assays. Three-week-old seedlings of the susceptible rice cultivar Oryza sativa cv. Nakdongbyeo were used to conduct leaf infection assays (Huh et al., 2017). Conidial suspensions $\left(5 \times 10^{4}\right.$ conidia/ $\mathrm{ml}$ ) of wild-type, mutant, and complemented strains collected from 10-day-old oatmeal medium were sprayed onto rice leaves and incubated in the dark for $24 \mathrm{~h}$, then moved to a growth chamber for a $16 \mathrm{~h}$ photoperiod with fluorescent light (Kim et al., 2014). Disease development was examined after 7 days of incubation. Appressorium penetration and hyphal infection assays were performed using dropping conidial suspensions $\left(5 \times 10^{4}\right.$ conidia/ $\mathrm{ml}$ ) or by placing agar plugs ( $6 \mathrm{~mm}$ in diameter) of wildtype, mutant, and complemented strains onto rice leaves incubated under the same conditions as the spray assay. Infected tissues of rice leaves were observed after 2 days of inoculation. Briefly, a conidial suspension $\left(5 \times 10^{4}\right.$ conidia/ $\mathrm{ml}$ ) was dropped on a rice sheath and incubated, then the invasive growth in plants was observed after 2 days.

\section{Results}

Phylogenetic analysis and domain structure of the Rho family. The $M$. oryzae genome contains six members of 


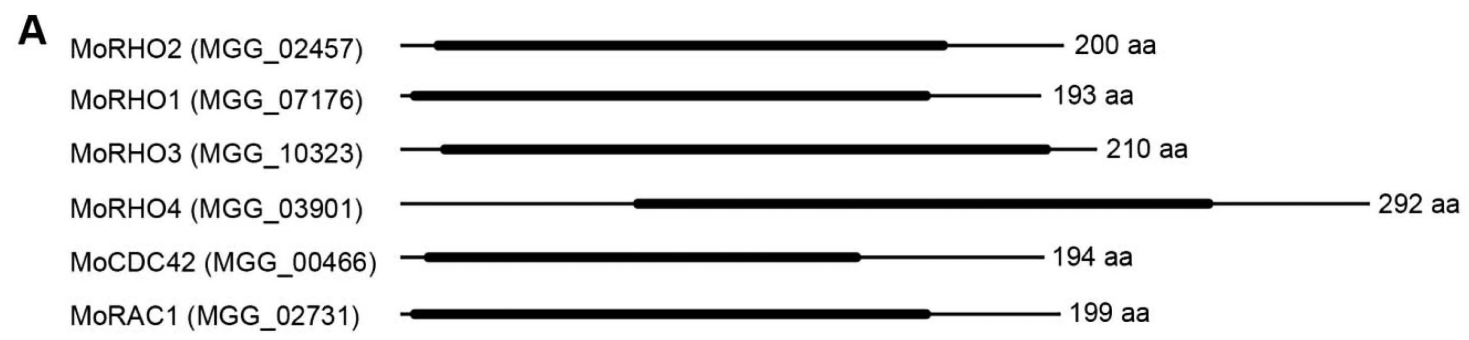

B

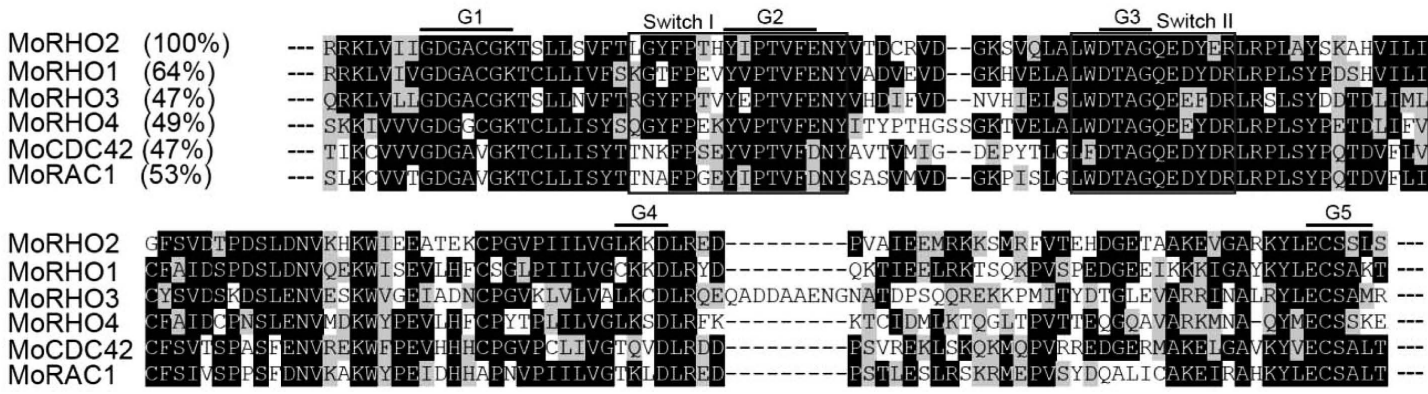

Fig. 1. Domain structure and conserved amino acids of RHO GTPase in M. oryzae. (A) Schematic structure of the small GTP-binding protein domain (IPR005225) in the RHO protein family. The domain structure was predicted using InterProScan. (B) The conserved amino acid sequence alignment of Rho GTPase. G1, G2, G3, G4, G5, switch I, and switch II denote special motifs in the small GTPbinding protein domain. The identity of each protein BLAST search with MoRHO2 is followed by its name.

the Rho family, which include MoRHO1 (MGG_07176), MoRHO2 (MGG_02457), MoRHO3 (MGG_10327), MoRHO4 (MGG_03901), MoCDC42 (MGG_00466), and MoRACl (MGG_02731). Each of the six protein sequences consists of one conserved domain named the small GTP-binding protein domain (IPR005225) (Fig. 1A). A BLASTP search of the NCBI database showed that MoRHO2 had 64\% identity with MoRHO1, 47\% identity with MoRHO3 and MoCDC42, 49\% identity with MoRHO4, and 53\% identity with MoRAC1 (Fig. 1B).

A phylogenetic tree was constructed to analyze the relationships among MoRHO2 and orthologs from other organisms (Fig. 2A). MoRHO2 was closely related to proteins from $C$. gloeosporioides, $F$. graminearum, $N$. crassa, S. sclerotiorum, and A. nidulans, but was genetically distant to other homologs from animal or plants. The small GTPase MoRHO2 shared 92\% sequence identity with CEF78997.1 from $F$. graminearum and 27\% sequence identity with related proteins from D. melanogaster (Fig. 2B). Together, these results indicate that $\mathrm{RHO} 2$ orthologs are well-conserved in fungi.

Expression and targeted gene deletion of MoRHO2. To order to predict functional roles of MoRHO2 in developmental stages of $M$. oryzae, the expressions of targeted genes were measured using qRT-PCR during the interaction of M. oryzae and its host plant. MoRHO2 was significantly expressed in conidia, appressoria, and invasive hyphae in host plants, but not in germinated conidia (Fig. 3A). Expression of MoRHO2 was greatly induced ( $>8$-fold) in infected host plants, raising the possibility that $\mathrm{MoRHO} 2$ may play a role in pre- and postinfection. Therefore, we generated a targeted gene deletion mutant to study the roles of $\mathrm{MoRHO} 2$ in fungal pathogenic development. The deletion mutant was generated by introducing a knockout construct selected through the selectable marker-hygromycin cassette (Fig. 3B). In addition, $\triangle M$ Morho 2 was further verified by Southern blotting and RT-PCR (Fig. 3C and D). To confirm that the phenotype of $\triangle M$ Morho 2 resulted from deletion of the targeted gene, the mutant was complemented with a genomic copy of MoRHO2. A complemented transformant (Morho2c) showed recovered expression of MoRHO2.

Appressorium formation on a hydrophobic surface. We evaluated the role of $\mathrm{MoRHO} 2$ gene in vegetative growth rate, conidiation, and conidium germination in $M$. oryzae by comparing phenotypes of the wild type, $\triangle M o r h o 2$, and Morho2c strains. The 4 Morho2 mutant exhibited no significant differences compared to the wildtype and Morho2c in vegetative growth, conidiation, and conidium germination, which indicated that $\mathrm{MoRHO} 2$ gene is not associated with these developmental stages (data not shown). Then we tested the role of MoRHO2 in 
A

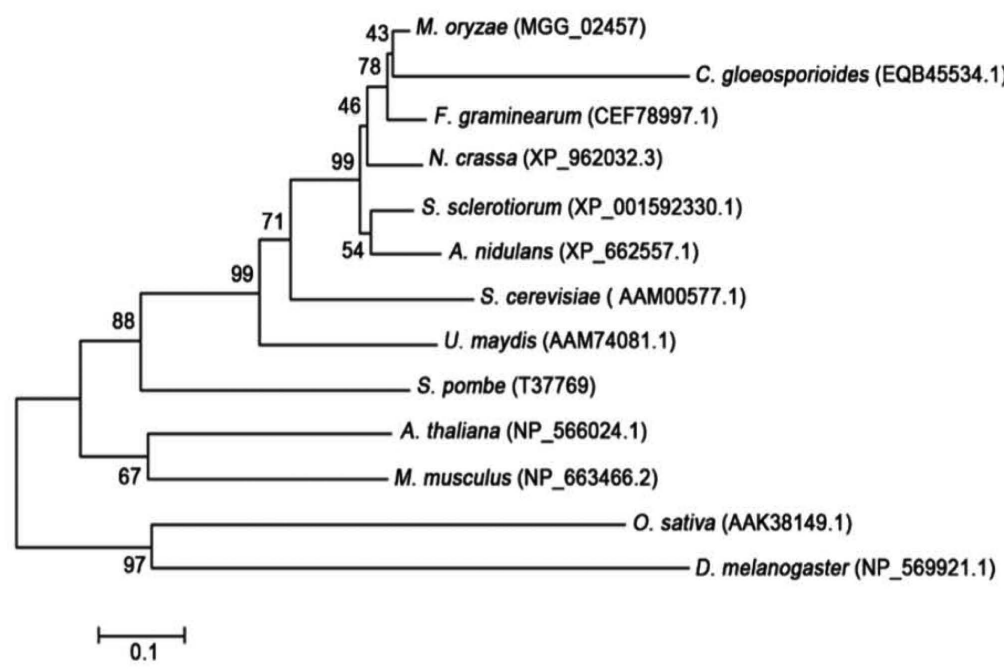

B

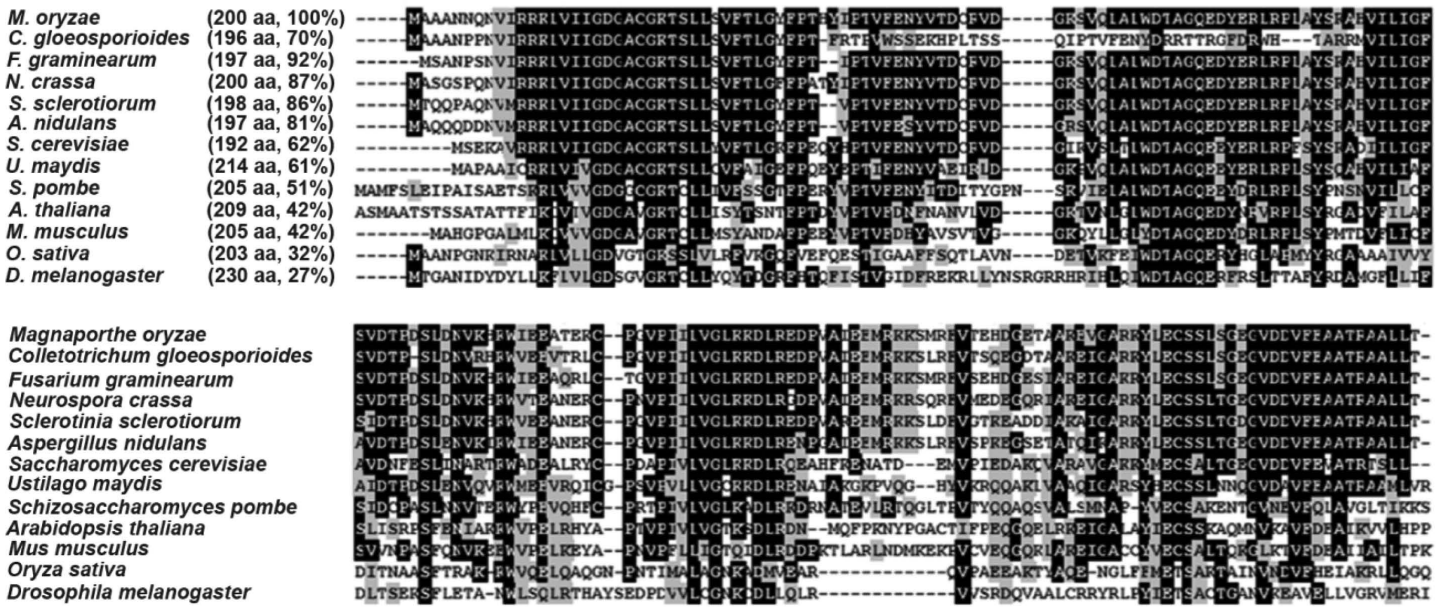

Fig. 2. Phylogenetic analysis and conserved amino acid sequence alignment of MoRHO2 and homologues from other organisms. (A) Phylogenetic analysis among MoRHO2 and homologues. A phylogenetic tree was generated using a neighbor-joining method based on comparing MoRHO2 and its homologues. (B) The conserved amino acid sequence alignment among MoRHO2 and homologues. The identity of each protein BLAST search with MoRHO2 is followed by its name.

appressorium development. When the conidial suspension was dropped onto hydrophobic coverslips, $92.6 \%$ and $99 \%$ of the wild type conidia generated appressoria at 8 and 48 hr, respectively (Fig. 4). Surprisingly, $\triangle M o r h o 2$ did not develop appressorium on hydrophobic surface at $8 \mathrm{hr}$, and only $6 \%$ at $48 \mathrm{hr}$. This result suggested that MoRHO2 is required for proper development of appressoria in response to the hydrophobic surface. Because $C$. gloeosporioides $R h o B$, a MoRHO2 orthologous, is involved in the cAMP signaling pathway (Xu et al., 2016), we further investigated the effects of exogenous cAMP on defective appressoria development in $\triangle M o r h o 2$. Notably, in the presence of cAMP, appressorium formation was restored in $\triangle M o r h o 2$ like wild type on hydrophobic surface. However, these appressoria were abnormally shaped, indicating that MoRHO2 is important for surface recognition and morphological regulation during appressorium development.

In a previous study, M. oryzae penetrated host cells through appressorium-like structure (ALS) developed on hyphal tips (Kong et al., 2013). Therefore, we examined whether MoRHO2 was involved in ALS development in M. oryzae. The $\triangle M o r h o 2$ mutant showed a delayed development of ALS with an abnormal shape from hyphal tips on the same hydrophobic surface. These results suggested that MoRHO2 is required for appressorium morphogenesis derived from conidia and hyphae.

Roles of $\mathrm{MoRHO} 2$ in pathogenic development. To understand the role of the MoRHO2 gene in disease 
A

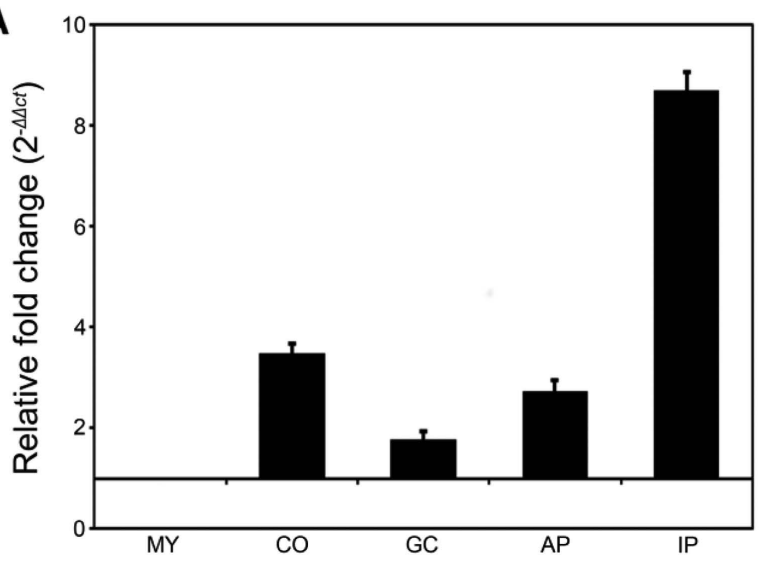

B

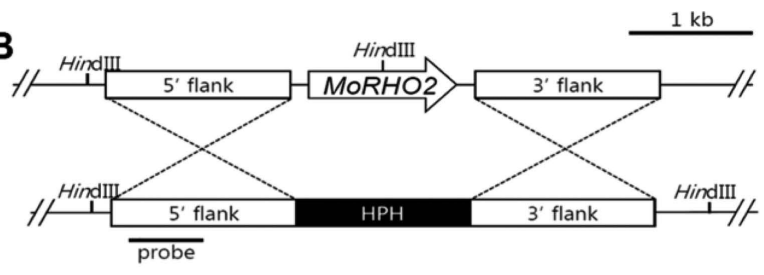

C

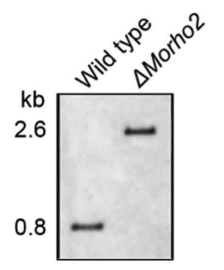

D

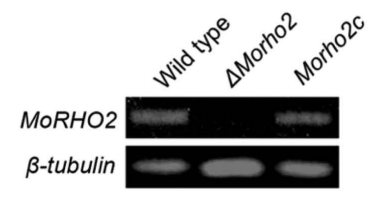

Fig. 3. The expression profile and targeted gene deletion of MoRHO2. (A) The expression profile of MoRHO2 in different developmental stages of M. oryzae. The expression of MoRHO2 was measured during five stages including mycelia (MY), conidia (CO), germinated conidia (GC), appressoria (AP), and infectious hyphae stages in rice leaves (IP). The results were normalized to $\beta$-tubulin and presented with a relative value of 1 in MY. (B) The targeted gene knockout of MoRHO2. The knockout strategy used the $H P H$ cassette to replace MoRHO2. (C) The conformation of the MoRHO2 deletion using southern blot analysis. The genomic DNA was digested with HindIII and hybridized with specific probes. (D) Reverse transcription-PCR was used to check the expression of MoRHO2. The total RNA was extracted from wild type, $\triangle M o r h o 2$, and Morho2c samples.

development, susceptible 3-week-old rice leaves were sprayed with conidial suspensions of wild type, $\triangle M o r h o 2$, and Morho2c. Treatment of wild type and Morho2c led to typical necrotic lesions (Fig. 6A). In contrast, $\triangle M$ Morho2 completely failed to cause disease symptoms. To further characterize the roles of MoRHO2 in penetration,

A

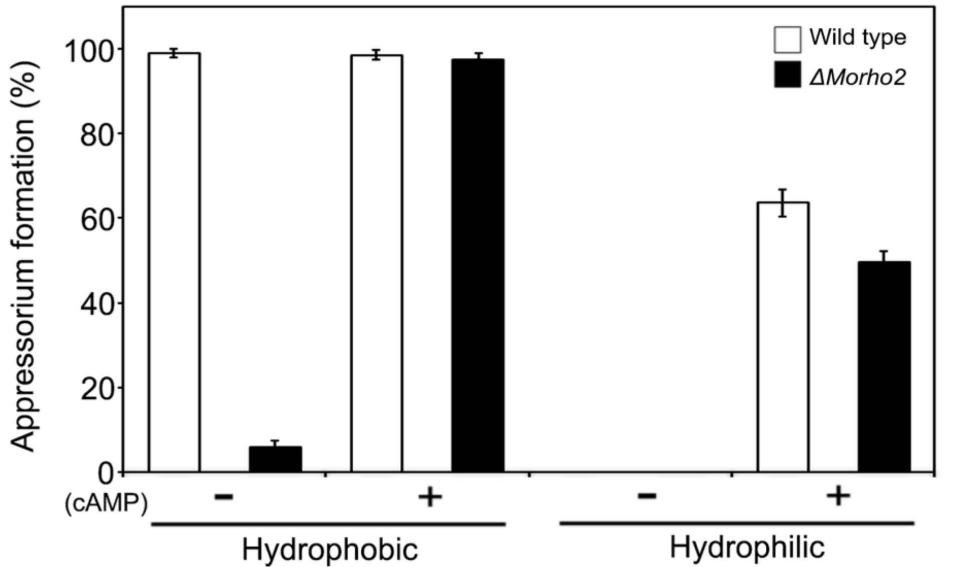

B

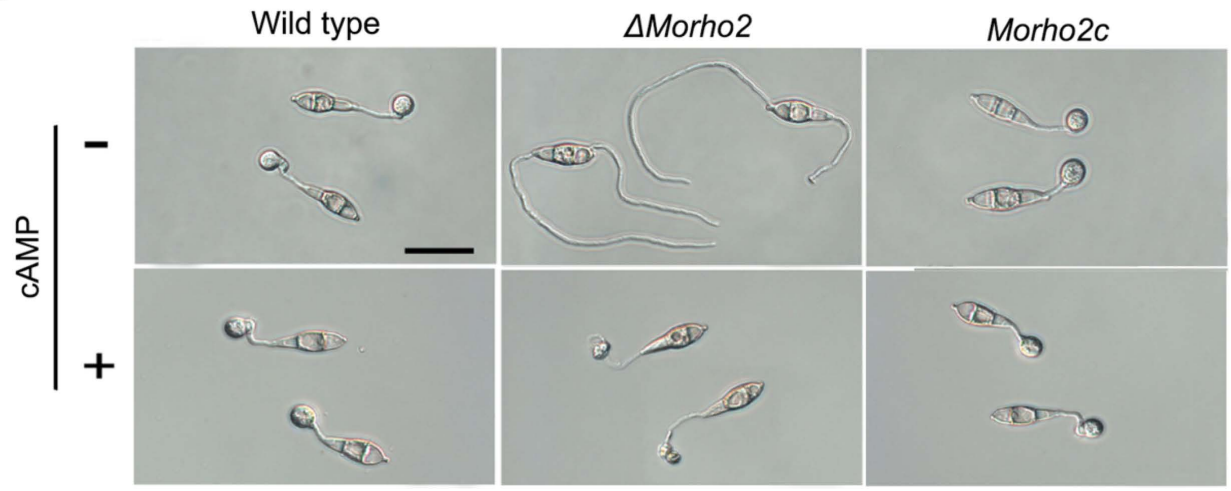

Fig. 4. Appressorium formation on artificial surfaces. (A) Statistical analysis of appressoria formed on the hydrophobic and hydrophilic surface. Appressorium formation was assessed at $48 \mathrm{~h}$ after inoculation. (B) The appressorium morphology on a hydrophobic surface. Appressoria were observed after a $6 \mathrm{~h}$ incubation. Scale bar $=20 \mu \mathrm{m}$. 


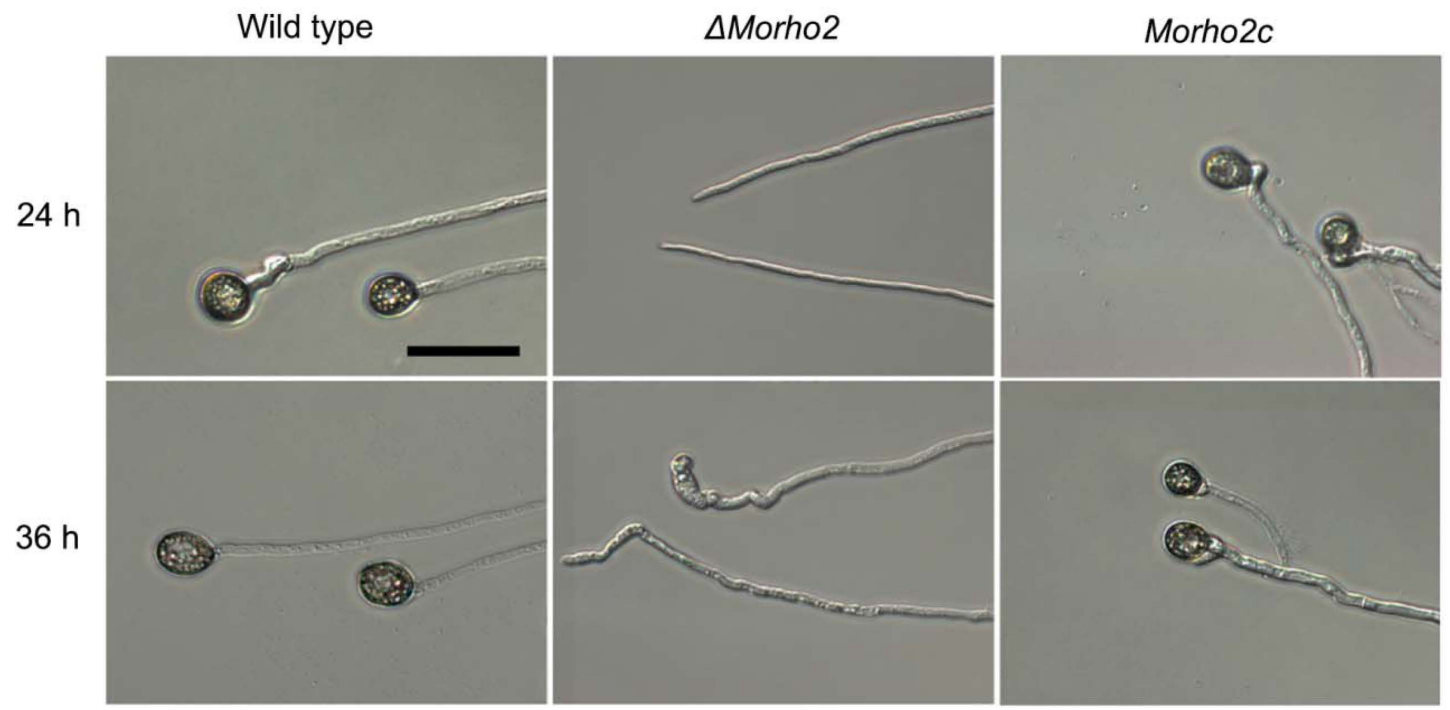

Fig. 5. An appressorium-like structure (ALS) formed on hyphal tips. Hyphal plugs (5 $\mathrm{mm}$ in diameter) of wild type, $\Delta M o r h o 2$, and Morho2c samples were placed on hydrophobic surfaces. Photographs were taken after 24 and $36 \mathrm{~h}$. Scale bar $=50 \mu \mathrm{m}$.

A

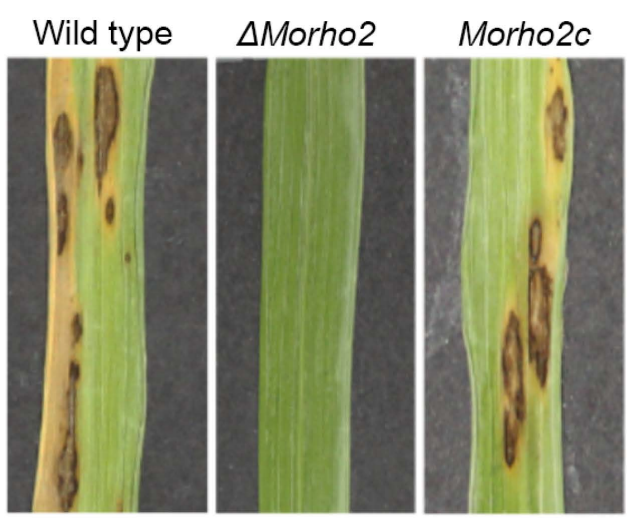

B

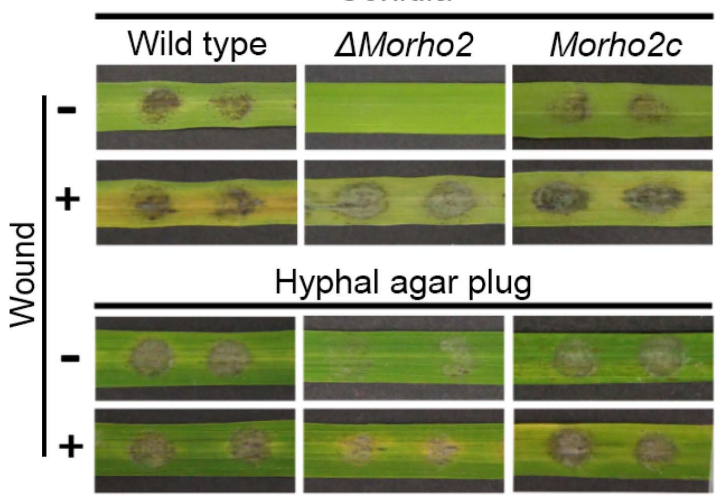

Fig. 6. Plant pathogenic assays. (A) The spray assays. The conidial suspension was sprayed onto rice leaves and the leaves were incubated for 7 days. (B) The influence of wounding on disease development. Conidial drops or hyphal plugs (6 $\mathrm{mm}$ in diameter) were inoculated onto rice leaves with or without wounding and the leaves were incubated for 2 days. conidial drops were inoculated onto detached wounded or unwounded rice leaves (Fig. 6B). Similar to the results from conidial spray assays, wild-type and Morho2c strains developed typical lesions on unwounded rice leaves, but $\Delta$ Morho2 did not cause any lesions. However, $\Delta$ Morho2 led to lesion development on wounded rice leaves, although they were very weak compared to those of wild type or Morho2c. When hyphal plugs were inoculated

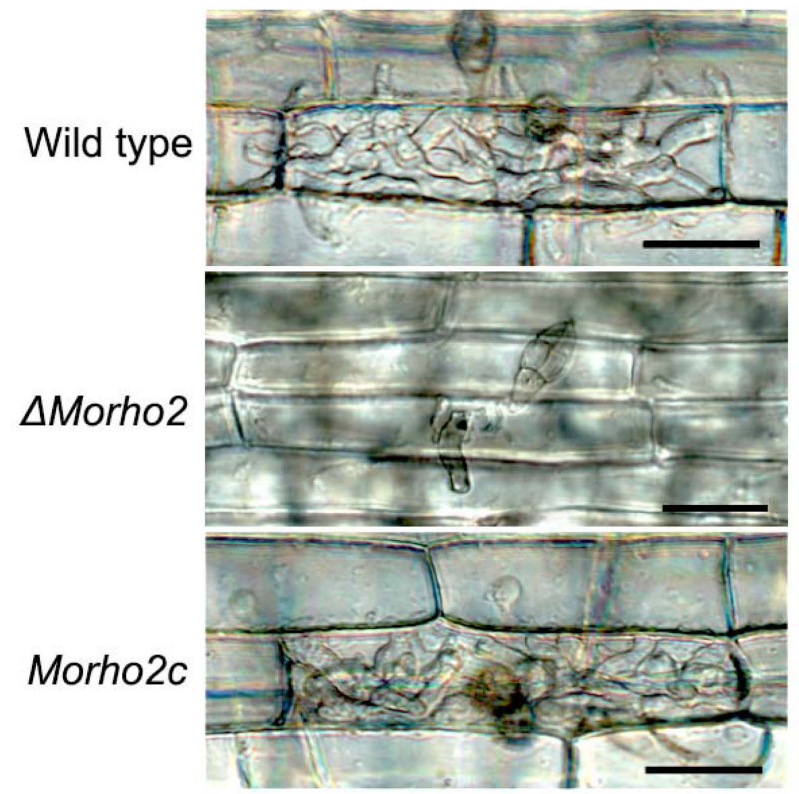

Fig. 7. Penetration assays. A conidial suspension of the indicated strains was dropped on rice sheath cells. Photographs were taken at 2 days after inoculation. Scar bar $=50 \mu \mathrm{m}$. 
onto detached rice leaves, $\triangle$ Morho 2 caused weaker disease symptoms on both wounded and unwounded rice leaves compared to those by the wild type and Morho2c. Together, these results suggested that MoRHO2 is required for full virulence in M. oryzae.

Appressorium penetration and invasive hyphal growth. To further characterize the association between $\mathrm{MoRHO} 2$ and disease development, we tested $\triangle M o r h o 2$ for penetration and invasive growth by inoculating a conidial suspension onto rice sheath cells. Similar to the defective appressorium development on artificial surfaces, conidia of $\Delta M o r h o 2$ were unable to develop appressoria on rice sheath cells, in contrast to those of the wild type and Morho2c. A small number of conidia $(<1 \%)$ in $\triangle M o r h o 2$ developed appressoria but these were morphologically abnormal and were unable to penetrate rice cells (Fig. 7). These results strongly suggest that $\mathrm{MoRHO2}$ is required for appressorium-mediated penetration in M. oryzae.

\section{Discussion}

M. oryzae is a model organism for studying fungal development and pathogenic development (Valent, 1990). In the present study, we showed that MoRHO2 plays an important role in $M$. oryzae preinfection-related development. MoRHO2 encodes a GTP-binding protein, which belongs to the Rho GTPase family (Dean et al., 2005). To characterize the functional role of MoRHO2 in M. oryzae, we generated a MoRHO2 deletion mutant and compared phenotypes during developmental stages in $M$. oryzae. The $\Delta$ Morho 2 mutant was unaffected in vegetative growth, conidiation, and conidial germination compared to the wild type, which indicates that $\mathrm{MoRHO} 2$ is not associated with these developmental stages.

The vast majority of $\triangle M o r h o 2$ conidia failed to produce appressoria on hydrophobic surface, indicating that MoRHO2 is required for appressorium formation in response to hydrophobicity (Fig. 4). Because the cAMP signaling pathway is well known in appressorium formation, we tested the effect of exogenous cAMP in the appressorium formation (Xu and Hamer, 1996). The appressorium formation rate in $\triangle M o r h o 2$ increased to a level similar to that wild type, but all of the $\triangle M o r h o 2$ appressoria exhibited an abnormal morphology, which suggests that $\mathrm{MoRHO2}$ is involved in the recognition of hydrophobicity for proper development of appressoria. However, the treatment of exogenous cAMP enabled $\triangle M o r h o 2$ mutant to bypass the signal recognition for appressorium development, but not for appressorium morphogenesis. It is noteworthy that the deletion of $C g R h o B$ in Colletotrichum gloeosporioides, a MoRHO2 ortholog, leads to the abnormal increase in cAMP levels, enhancement in appressorium development and alteration in cell wall integrity (Xu et al., 2016). Therefore, it is reasonable to hypothesize that MoRHO2 is involved in regulating cAMP level for appressorium development in M. oryzae, as supported with restoration of appressorium development with exogenous cAMP treatment in $\triangle M o r h o 2$ (Fig. 4). However, the mechanistic function of MoRHO2 in the cAMP signaling pathway is still unknown. M. oryzae develops an appressorium-like structure (ALS) on hyphal tips (Kong et al., 2013). Similar to abnormal appressoria on conidial germ tubes, the $\triangle M o r h o 2$ mutant also developed morphologically abnormal ALS on hyphal tips (Fig. 5). These data indicate that MoRHO2 is essential for appressorium morphogenesis on tips of conidial germ tubes and hyphae tips. Interestingly, the $\triangle C g R h o B$ mutant exhibited shorter germ tubes, reduction in conidial germination while the $\triangle M o r h o 2$ mutant was normal in germ tube formation and conidial germination, suggesting divergent roles of $\mathrm{RHO} 2$ orthologs in fungal pathogens (Xu et al., 2016).

Abnormal appressoria morphology in response to exogenous cAMP in the $\triangle$ Morho 2 mutant may suggest a defect in turgor pressure. This is supported by the lack of symptom development in both spray assay and sheath assay (Fig. 6). Interestingly, other studies have reported roles of MoRHO2 orthologs in the cell integrity in A. niger, F. graminearum, and $N$. crassa (Kwon et al., 2011; Richthammer et al., 2012; Zhang et al., 2013). The importance of cell wall integrity in maintaining turgor pressure of appressoria in M. oryzae is well documented (Xu et al., 1998). Moreover, in the budding yeast Saccharomyces cerevisiae, Rho2 affects Slt2, a mitogen-activated protein kinase (MAPK), through protein kinase $\mathrm{C}$, thus controlling cell integrity including ionic homeostasis and cell wall maintenance (Matia-González and Rodríguez-Gabriel, 2011). Considering that Mps1, a Slt2 ortholog, is associated with cell wall integrity in M. oryzae, we speculate that $\mathrm{RHO} 2$ may affect the regulation of Mps1 for cell wall integrity (Xu et al., 1998).

In summary, we characterized the role of MoRHO2 gene in $M$. oryzae morphogenesis and disease development. Rho GTPases are involved in essential molecular signaling pathways regulating pathogenicity in fungal species. MoRHO2 gene was involved in preinfection-related development of M. oryzae, including appressorium development and morphogenesis, and invasive growth inside host plant cells. Overall, our results provide a functional basis for $\mathrm{MoRHO2}$-mediated pre-infection development in $M$. 
oryzae.

\section{Acknowledgement}

This study was supported by Basic Science Research Program through the National Research Foundation of Korea grant (NRF-2013R1A1A1008444) funded by the Ministry of Education, Science and Technology, and by a grant (117035-03-2-SB010) funded by Export Promotion Technology Development Program, Ministry of Agriculture, Food and Rural Affairs, Republic of Korea.

\section{References}

An, B., Li, B., Qin, G. and Tian, S. 2015. Function of small GTPase Rho3 in regulating growth, conidiation and virulence of Botrytis cinerea. Fungal Genet. Biol. 75:46-55.

Arellano, M., Coll, P. M. and Pérez, P. 1999. RHO GTPases in the control of cell morphology, cell polarity, and actin localization in fission yeast. Microsc. Res. Tech. 47:51-60.

Choi, J., Kim, K. S., Rho, H. S. and Lee, Y. H. 2011. Differential roles of the phospholipase $\mathrm{C}$ genes in fungal development and pathogenicity of Magnaporthe oryzae. Fungal Genet. Biol. 48:445-455.

Dean, R. A. 1997. Signal pathways and appressorium morphogenesis. Annu. Rev. Phytopathol. 35:211-234.

Dean, R. A., Talbot, N. J., Ebbole, D. J., Farman, M. L., Mitchell, T. K., Orbach, M. J., Thon, M., Kulkarni, R., Xu, J.-R., Pan, H., Read, N. D., Lee, Y.-H., Carbone, I., Brown, D., Oh, Y. Y., Donofrio, N., Jeong, J. S., Soanes, D. M., Djonovic, S., Kolomiets, E., Rehmeyer, C., Li, W., Harding, M., Kim, S., Lebrun, M.-H., Bohnert, H., Coughlan, S., Butler, J., Calvo, S., Ma, L.-J., Nicol, R., Purcell, S., Nusbaum, C., Galagan, J.E. and Birren, B. W. 2005. The genome sequence of the rice blast fungus Magnaporthe grisea. Nature 434:980-986.

Du, Y., Shi, Y., Yang, J., Chen, X., Xue, M., Zhou, W. and Peng, Y.-L. 2013. A serine/threonine-protein phosphatase PP2A catalytic subunit is essential for asexual development and plant infection in Magnaporthe oryzae. Curr. Genet. 59:33-41.

Etienne-Manneville, S. and Hall, A. 2002. Rho GTPases in cell biology. Nature 420:629-635.

Guest, G. M., Lin, X. and Momany, M. 2004. Aspergillus nidulans RhoA is involved in polar growth, branching, and cell wall synthesis. Fungal Genet. Biol. 41:13-22.

Hamer, J. E. and Talbot, N. J. 1998. Infection-related development in the rice blast fungus Magnaporthe grisea. Curr. Opin. Microbiol. 1:693-697.

Han, J. H., Lee, H. M., Shin, J. H., Lee, Y. H. and Kim, K. S. 2015. Role of the MoYAK1 protein kinase gene in Magnaporthe oryzae development and pathogenicity. Environ. Microbiol. 17:4672-4689.

Hanna, S. and El-Sibai, M. 2013. Signaling networks of Rho GTPases in cell motility. Cell. Signal. 25:1955-1961.
Huh, A., Dubey, A., Kim, S., Jeon, J. and Lee, Y. H. 2017. Mo$J M J 1$, encoding a histone demethylase containing JmjC domain, is required for pathogenic development of the rice blast fungus, Magnaporthe oryzae. Plant Pathol. J. 33:193-205.

Karnoub, A. E., Symons, M., Campbell, S. L. and Der, C. J. 2004. Molecular basis for Rho GTPase signaling specificity. Breast Cancer Res. Treat. 84:61-71.

Kim, H. J., Han, J. H., Kim, K. S. and Lee, Y. H. 2014. Comparative functional analysis of the velvet gene family reveals unique roles in fungal development and pathogenicity in Magnaporthe oryzae. Fungal Genet. Biol. 66:33-43.

Kim, K. S. and Lee, Y. H. 2012. Gene expression profiling during conidiation in the rice blast pathogen Magnaporthe oryzae. PLoS ONE 7:e43202.

Kim, S., Park, S. Y., Kim, K. S., Rho, H. S., Chi, M. H., Choi, J., Park, J., Kong, S., Park, J., Goh, J. and Lee, Y. H. 2009. Homeobox transcription factors are required for conidiation and appressorium development in the rice blast fungus Magnaporthe oryzae. PLoS Genet. 5:e1000757.

Kong, L. A., Li, G. T., Liu, Y., Liu, M. G., Zhang, S. J., Yang, J., Zhou, X. Y., Peng, Y. L. and Xu, J. R. 2013. Differences between appressoria formed by germ tubes and appressoriumlike structures developed by hyphal tips in Magnaporthe oryzae. Fungal Genet. Biol. 56:33-41.

Kwon, M. J., Arentshorst, M., Roos, E. D., van den Hondel, C. A., Meyer, V. and Ram, A. F. 2011. Functional characterization of Rho GTPases in Aspergillus niger uncovers conserved and diverged roles of Rho proteins within filamentous fungi. Mol. Microbiol. 79:1151-1167.

Madaule, P. and Axel, R. 1985. A novel ras-related gene family. Cell 41:31-40.

Mahlert, M., Leveleki, L., Hlubek, A., Sandrock, B. and Bölker, M. 2006. Rac1 and Cdc42 regulate hyphal growth and cytokinesis in the dimorphic fungus Ustilago maydis. Mol. Microbiol. 59:567-578.

Martínez-Rocha, A. L., Roncero, M. I., López-Ramirez, A., Mariné, M., Guarro, J., Martínez-Cadena, G. and Di Pietro, A. 2008. Rho1 has distinct functions in morphogenesis, cell wall biosynthesis and virulence of Fusarium oxysporum. Cell. Microbiol. 10:1339-1351.

Matia-González, A. M. and Rodríguez-Gabriel, M. A. 2011. Slt2 MAPK pathway is essential for cell integrity in the presence of arsenate. Yeast 28:9-17.

Nakano, K., Mutoh, T., Arai, R. and Mabuchi, I. 2003. The small GTPase Rho4 is involved in controlling cell morphology and septation in fission yeast. Genes Cells 8:357-370.

Park, J., Park, B., Jung, K., Jang, S., Yu, K., Choi, J., Kong, S., Park, J., Kim, S. Kim, H., Kim, S., Kim, J. F., Blair, J. E., Lee, K., Kang, S. and Lee, Y. H. 2007. CFGP: a web-based, comparative fungal genomics platform. Nucleic Acids Res. 36:D562-D571.

Rasmussen, C. G. and Glass, N. L. 2005. A Rho-type GTPase, rho-4, is required for septation in Neurospora crassa. Eukaryot. Cell 4:1913-1925. 
Richthammer, C., Enseleit, M., Sanchez-Leon, E., März, S., Heilig, Y., Riquelme, M. and Seiler, S. 2012. RHO1 and RHO2 share partially overlapping functions in the regulation of cell wall integrity and hyphal polarity in Neurospora crassa. Mol. Microbiol. 85:716-733.

Ridley, A. J. 2001. Rho family proteins: coordinating cell responses. Trends Cell Biol. 11:471-477.

Ryder, L. S. and Talbot, N. J. 2015. Regulation of appressorium development in pathogenic fungi. Curr. Opin. Plant Biol. 26:8-13.

Saleh, D., Milazzo, J., Adreit, H., Fournier, E. and Tharreau, D. 2014. South-East Asia is the center of origin, diversity and dispersion of the rice blast fungus, Magnaporthe oryzae. New Phytol. 201:1440-1456.

Schaefer, A., Reinhard, N. R. and Hordijk, P. L. 2014. Toward understanding RhoGTPase specificity: structure, function and local activation. Small GTPases 5:e968004.

Smithers, C. C. and Overduin, M. 2016. Structural mechanisms and drug discovery prospects of Rho GTPases. Cells 5:26.

Talbot, N. J., Ebbole, D. J. and Hamer, J. E. 1993. Identification and characterization of $M P G 1$, a gene involved in pathogenicity from the rice blast fungus Magnaporthe grisea. Plant Cell 5:1575-1590.

Valent, B. 1990. Rice blast as a model system for plant pathology. Phytopathology 80:33-36.

Vasara, T., Salusjärvi, L., Raudaskoski, M., Keränen, S., Penttilä, M. and Saloheimo, M. 2001. Interactions of the Trichoderma reesei rho 3 with the secretory pathway in yeast and $T$. reesei. Mol. Microbiol. 42:1349-1361.

Wittinghofer, A. and Vetter, I. R. 2011. Structure-function relationships of the G domain, a canonical switch motif. Annu.
Rev. Biochem. 80:943-971.

Xu, J. R. and Hamer, J. E. 1996. MAP kinase and cAMP signaling regulate infection structure formation and pathogenic growth in the rice blast fungus Magnaporthe grisea. Genes Dev. 10:2696-2706.

Xu, J. R., Staiger, C. J. and Hamer, J. E. 1998. Inactivation of the mitogen-activated protein kinase Mps1 from the rice blast fungus prevents penetration of host cells but allows activation of plant defense responses. Proc. Natl. Acad. Sci. U.S.A 95:12713-12718.

Xu, X., Wang, Y., Tian, C. and Liang, Y. 2016. The Colletotrichum gloeosporioides RhoB regulates cAMP and stress response pathways and is required for pathogenesis. Fungal Genet. Biol. 96:12-24.

Yu, J. H., Hamari, Z., Han, K. H., Seo, J. A., Reyes-Domínguez, Y. and Scazzocchio, C. 2004. Double-joint PCR: a PCR-based molecular tool for gene manipulations in filamentous fungi. Fungal Genet. Biol. 41:973-981.

Zhang, C., Wang, Y., Wang, J., Zhai, Z., Zhang, L., Zheng, W., Zheng, W., Yu, W., Zhou, J., Lu, G., Shim, W. B. and Wang, Z. 2013. Functional characterization of Rho family small GTPases in Fusarium graminearum. Fungal Genet. Biol. 61:9099.

Zheng, W., Chen, J., Liu, W., Zheng, S., Zhou, J., Lu, G. and Wang, Z. 2007. A Rho3 homolog is essential for appressorium development and pathogenicity of Magnaporthe grisea. Eukaryot. Cell 6:2240-2250.

Zhu, L., Zhu, J., Liu, Z., Wang, Z., Zhou, C. and Wang, H. 2017. Host-Induced Gene Silencing of Rice Blast Fungus Magnaporthe oryzae Pathogenicity Genes Mediated by the Brome Mosaic Virus. Genes 8:241. 